\title{
Development of Analysis Methods in Operating Systems
}

\author{
Aaron Don M. Africa, Floyd Robert C. Dim, Sandra Margarita L. Fernando, \\ Daniel Lester C. Guillermo, Leila Yasmin C. Young \\ De La Salle University, Manila \\ 2401 Taft Ave., Malate, Manila 1004, \\ Philippines, aaron.africa@dlsu.edu.ph
}

\begin{abstract}
The windows operating system is the primary user interface developed by Microsoft. This serves as a user's main interface when transferring or traversing through files as well as run programs. Each updated version of Windows comes with new menu designs and ideally a better performance than the previous versions due to better optimization. To observe these changes, Windows 7, 8 and 10 , the 3 most recent versions, have been tested by measuring each of their boot-up time and performances. Results verify that Windows 10 has a faster boot-up time and better performance compared to the previous versions.
\end{abstract}

Key words: Operating System, User interface, Performance, Optimization, Windows.

\section{INTRODUCTION}

The operating system is one of the major foundations of technology today, and just as other devices are being developed constantly, the operating system is also being developed constantly to be able to handle all the new applications and programs that are being developed with every new release. Microsoft is one of the well-known developers of the operating system, specifically the Windows operating system. Other companies that developed their own operating systems are Apple with MacOS, and Canonical Ltd. with Ubuntu (a complete Linux operating system). This paper will focus mainly on the timeline of Microsoft's Windows OS and how the company has improved its operating systems throughout the years of development and development of this widely used operating system.

Microsoft started with the Microsoft Disk Operating System or DOS. This was developed for International Business Machine-compatible computers for personal use. The early versions of the Disk Operating System were fairly simple until further versions became more complex and incorporated other features of operating systems. This was first developed in 1981 called the MS-DOS. Four years later, the first version of the Windows Operating System was introduced to the public. The operating system was dubbed "Windows" because it represents the boxes or windows aspect of the operating system. Windows 1.0 utilized the mouse and enabled users to point and click to access windows, rather than typing commands with the previous Microsoft Disk Operating System. With the release of Windows 3.0 onwards the operating system began to gain color and better icons for a user-friendly interface. This version of Windows also introduced file management as well as games such as minesweeper and solitaire that still comes with the recent Windows Operating Systems today. Come 1995, Microsoft launched Windows 95, with a new user interface and better program allocation. This version of Windows supports 32-bit apps [1]. From there, Windows OS continued to develop into the kind of operating system the majority of the population use today.

\section{BACKGROUND OF THE STUDY}

The Windows operating system was developed to cater to the initially growing technological industry were different kinds of devices were invented and introduced to the public to introduce a new way of file management especially in big companies like a bank. The operating system began from just using a keyboard to input commands with the initial Disk Operating System or DOS then Microsoft developed their Windows OS to a more interactive interface. The very first version of windows utilized the mouse, making the input of commands quicker with a simple click. It also makes accessing files faster since users need only to point and click.

Further development of the Windows Operating System introduced icons, file management, program management, printing management, and many others. This is also the point in the Windows Operating System's timeline where the usual games that come with a Windows Operating System were added like Minesweeper and Solitaire. It was in the late 1990s where the Windows Operating System began to take shape into the kind of Windows the majority of the population use. Inclusion of the 32bit support began and processing of applications became faster. The initial Disk Operating System was almost virtually removed from the Windows Operating System since Windows became much more complex than the Disk Operating System. Come the year 2000, DOS was 
removed completely from the Windows Operating System as the operating system can reboot itself without the need to go into DOS mode. Further, development leads to a business centered operating systems where the inclusions of these were the basics of an office and an office workplace. Very recent versions of Windows, namely Windows 7 , Windows 8 , and Windows 10, took technological advancements in mind, with a touch screen option available as well as a more sophisticated minimalist "Start Menu" with the considerations of a user-customized interface.

\section{STATEMENT OF THE PROBLEM}

This paper will discuss the user interface of Windows that has been developed starting from 1985. This user interface has been one of the most popular user interfaces as it is more user-friendly as compared to the other interfaces. Throughout the development of the Window's user interface, features are developed so that it could enhance the user's experience of using their product. These updates are essential so that the computer could keep up to date, fill the holes of possible virus attacks, and allow the user to experience faster processing speed. However, not all of the Windows user interfaces have given users satisfaction. For instance, many Windows users did not find the Windows 8 user interface as a satisfying update. This is because some features were difficult to understand for new users. It would be hard for new users to explore the new Windows user interface. Despite the complaints, there are also positive reviews where the new Windows 8 can process data faster as compared to the previous user interface. In line with this, knowing how the Windows user interface developed, and the advantages and disadvantages of each update will allow the readers to understand the importance of each generation despite the possible setbacks.

\section{SIGNIFICANCE OF THE STUDY}

The main idea of the study is to be able to learn about the user interface that a computer uses to allow the user to perform tasks involving computers. By learning about how to navigate through a user interface properly, the user can perform the tasks needed efficiently and correctly. Another main idea of the study is to be able to observe how the user interface is affecting the resources of the computer. What this means is that by using a certain user interface, the usage of parts of the computer, such as the random-access memory and the central processing unit, may be higher than using a different user interface. To test this, the user interfaces that will be compared to each other is Windows. Each version of Windows has been out for a long time, so comparing how they work, how they use computer resources and how people think of them in terms of features, usability and the resource consumption between them. The group also wants to test the boot times of each version of Windows that are relevant to modern computing as boot times also affect the experience that the user has while navigating through the user interface. These points are what the group wants to tackle in the study.

\section{DESCRIPTION OF THE SYSTEM}

Windows serves as the primary operating system developed by Microsoft. This provides a graphical interface that allows users to traverse through different files and data types. The interface contains various menus, buttons and designs which allows the user to understand how to browse each menu. Systems in the interface run simultaneously to be able to receive data from the user, process those data internally. The system was made to be able to run only on a specific type of hardware which it is compatible with. The performance of a system to do its task depends on the type of hardware it is running on as well as the efficiency of the data processing.

\section{METHOLOGY}

Windows serves as the primary operating system developed by Microsoft. This provides a graphical interface that allows users to traverse through different files and data types. The interface contains various menus, buttons, and designs which allows the user to understand how to browse each menu. Systems in the interface run simultaneously to be able to receive data from the user, process those data internally. The system was made to be able to run only on a specific type of hardware which it is compatible with. The performance of a system to do its task depends on the type of hardware it is running on as well as the efficiency of the data processing.

\section{REVIEW OF RELATED LITERATURE}

\subsection{User Interface}

A user interface is a channel for users to connect with a certain device. This system is designed to relay information between the user input to devices the user interacts with. These devices include monitors or display screens, mouse, and keyboard [2]. The user interface is not only limited to computer devices. This can also be seen in other applications such as toys, virtual reality, neuroinflammatory responses, navigation, integration assistants [3], FPGA and alarm systems [4,5].

In recent years, toys, used for leisure and social activities, have integrated hardware as well as software for better user experience. These toys are known as smart toys for the integration of software into the toy's hardware. Note that smart toys are considered to be user interfaces because of the exchange of information between user input and output. They conclude that Toy-User-Interfaces (ToyUI), can be beneficial for future researchers, designers, end-users, as well as therapists and educators [6].

Some technologies today are not user-friendly, convenient, comfortable, and efficient. Many users note that the results from these technologies are not accurate because of the varying parameters, or it is not an effective teaching tool for students to visualize concepts. However, through virtual 
reality (VR) technologies, interactions between users and the device helped many users find solutions to their problems. For instance, in the field of rehabilitation, testers could identify the source of discomfort and the appropriate ergonomic solutions for each patient. This VR-based interface makes use of games to identify the needs of each patient. This developed interface makes use of Unity3D and Kinect motion sensor as the user's input. Though the authors note that the exoskeleton of the devices can be further improved [7]. Another instance is where the VR interface is used as an alternative to traditional architectural courses. Though the experience was limited to certain groups of people, each group was able to visualize the model presented by their professors [8]. The VR interface may have different performances depending on the parameters used to develop the VR. For instance, it is easier for a user to learn when the VR uses a speech or 2D interface, while a $3 \mathrm{D}$ interface would be more fun and enjoyable for users $[9,10]$.

In the field of neuroscience, it is possible to have neuroinflammation as a result of brain-implanted devices. Many researchers have developed new devices and strategies to reduce the trauma and neuroinflammation that could occur. One way to reduce this is to have a frequent metric published that can be quantified so that it could be analyzed by the researchers and doctors. A graphical user interface may be used to analyze and monitor the immunohistochemistry images of neuroinflammation. And the results of using a graphical user interface was proved to be valid with previous publications on the topic. Moreover, the use of this interface is very convenient, and it provided results that fell within the region of interest $[11,12]$.

Other than academic and medicinal uses for user interfaces, these can also be applied in the field of tourism. A user interface was designed for tourists to engage and experience the cultural attractions found in Italy. This is done by giving the user a mid-air visits many attractions such as domes, fortified farms, caves, and many more. The user gets immersed in this virtual environment by identifying the gestures the user creates to explore the environment $[13,14,15,16]$. This is an effective tool for tourism because it creates a mental image of a tourist spot for tourists. Through a VR interface, tourists have an idea of the tourist spot prior to visiting the tourist spot physically [17].

\subsection{Computer Performance}

Computer systems can be defined by their performance to process data through a metric system. This system allows the system to evaluate itself and reconstruct its structure. Moreover, computer performance can be defined as a set of algorithms that evaluates system behavior [18]. Depending on the device's purpose, computers can compute large amounts of data to improve computational power. According to one study, computers can be grouped into clusters so that they can perform better $[19,20]$. Though a computer's performance can be improved in different ways. For instance, information systems and computer self-efficacy contribute to the enhancement of a computer's overall performance [21,22, 23].

\subsection{Optimization}

Optimization is defined to be a system to fully use its resources effectively. These can be seen in any system today. For instance, it can be used as a tool for diagnosis [24], thermodynamic cooling [25], finance [26], biosystems [27], neuroscience [28], and structural engineering [29]. There is also an algorithm that is used for the optimization of global problems called the Selfish Herds Optimizer (SHO). This algorithm mimics the behavior of a selfish herd that avoids predators. Moreover, a set of evolutionary operators that revolve around the selfish herd theory. This algorithm was proved to give competitive results to global optimization in comparison to other algorithms [30]. This algorithm also has Control System feedback characteristics [31,32].

\section{THEORETICAL CONSIDERATIONS}

\subsection{Spatial imaging}

The run time and performance of a system may also depend on its spatial imaging. To expound, a system with more assets in its interface may take a longer time to run as to an interface with lesser asset images. Examples of these assets are from the sounds when various buttons are clicked, image design of the various menus, or the effects when switching windows.

\subsection{Rough Set Theory}

Rough set theory is utilized when monitoring the current performance of a system at a given time. This is more prominent in the task manager. The amount of memory the computer is using is represented in megabytes. This is not the exact number of the current memory but only a rough estimated value [33].

\subsection{Electronic Sensors}

Various electronic sensors may also affect the performance of a system based on the amount of data being transferred from that sensor. The larger the input data, the longer the time required to process such data. An example of this is data that records an image of an area that would be much larger than a keyboard that only sends simpler data types [34,35].

\subsection{Artificial Neural Network}

A computer is capable of selecting the fastest way to analyze data by simplifying it, this will improve the computer's performance as it does not need to process repeated data.

\subsection{Database Monitoring}

The size of the database will affect the speed in which the computer boots up as it has to process each one to allow the accessibility of each type of data when the user requires it. 
Aaron Don M. Africa et al., International Journal of Emerging Trends in Engineering Research, 8(5), May 2020, 1926 - 1931

\subsection{Data/ Information Transfer}

The performance relies on the amount of time for data or information to be transferred around in a computer's system. When the transfer of data is inefficient, the performance of the computer to do a task is hindered and is slowed down as well.

\section{DATA AND RESULTS}

Each boot-up time for windows has been recorded 3 times and is averaged. Below are the results of the 9 tests performed on Windows 7, 8 and 10. These are the time it takes from the press of the power button to the main UI screen. These tests were performed on a Lenovo Idea pad 320. Tables 1, 2 and 3 shows the boot up attempts in Windows 7, 8 and 10 respectively.

Table 1: Three Windows 7 boot-up time attempts

\begin{tabular}{|l|l|}
\hline First Attempt & 34.89 seconds \\
\hline Second Attempt & 33.22 seconds \\
\hline Third Attempt & 35.64 seconds \\
\hline Average time & 34.58 seconds \\
\hline
\end{tabular}

Table 2:Three Windows 8 boot-up time attempts

\begin{tabular}{|l|l|}
\hline First Attempt & 28.35 seconds \\
\hline Second Attempt & 29.74 seconds \\
\hline Third Attempt & 28.12 seconds \\
\hline Average time & 28.74 seconds \\
\hline
\end{tabular}

Table 3:Three Windows 10 boot-up time attempts

\begin{tabular}{|l|l|}
\hline First Attempt & 26.86 seconds \\
\hline Second Attempt & 27.51 seconds \\
\hline Third Attempt & 25.14 seconds \\
\hline Average time & 26.5 seconds \\
\hline
\end{tabular}

Figure 1 shows the Bar Graph of the 3 average attempts in Windows 7,8 and 10.

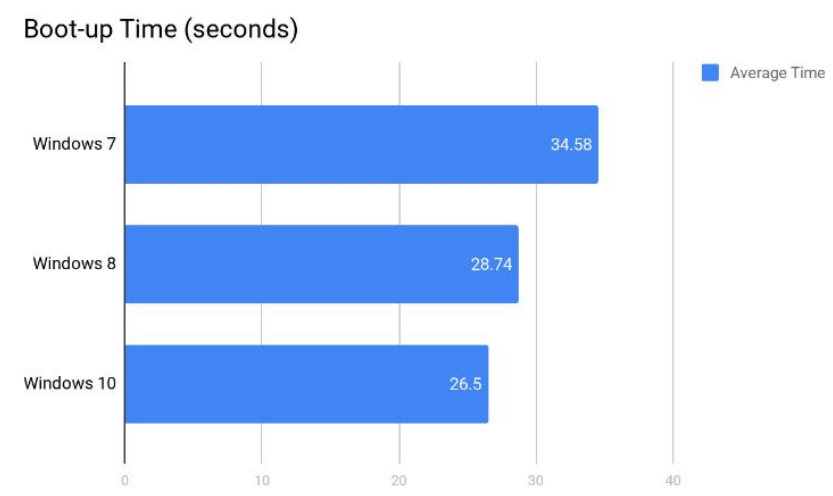

Figure 1:Bar graph of the 3 average times for windows 7,8 and 10

\section{ANALYSIS OF DATA}

Results of the data present that Windows 10 has the fastest boot-up time followed by Windows 8 then 7 . The boot-up time for Windows 7 is 34.89 seconds for the first, 33.22 seconds for the second, 35.64 seconds for the third and averages to 34.58 seconds. Windows 8 boot-up time is 28.35 seconds for the first, 29.74 seconds for the second, 28.12 seconds for the third and averages to 28.74 seconds. And lastly, Windows 10 boot-up time is 26.86 seconds for the first, 27.51 seconds for the second, 25.14 seconds and 26.5 seconds when averaged. These data affirm that as the generation of windows progresses, so does the efficiency of the software in configuring its data and settings when powered on.

As for the performances of each version of windows, existing data is available as a result of the tests of [32]. In this test, a game "Shadow of Mordor" was running in the 3 windows versions. Results show that for windows 7, the game ran at 52.2 frames per second, for windows 8 , the game ran at 52.1 frames per second and windows 10 ran at 52.4 frames per second. These results confirm that the most recent generation performs better than the previous ones. In overall speed and performance, windows 10 tops the previous versions as proven by the data.

\section{CONCLUSION}

To conclude, the Windows Operating System continues to develop into more complicated and sophisticated designs that are more or less customizable by the user for a more personalized look and feel to a user's preference. The Windows Operating System also develops outside of the personal computer, bringing the Windows Operating System to today's handheld devices pave the way for users to have a more portable workplace. Similar to Apple and their Apple ID, Windows and its mobile devices can now share files and information from one Windows device to another through a cloud service much like iCloud. 
The Windows Operating System transcends from the personal computer and begins to cater to the different kinds of users within the population. Businessmen, the youth, writers, artists, gamers can choose from the wide variety of Windows Operating Systems available that can cater to the kind of user experience they are looking for. The Windows Operating System continues to be versatile as well. During the time of the release of Windows 7, some apps or programs could only be run on a Windows XP or Vista; now with Windows 10, the development of this version of Windows takes into consideration the compatibility of the recent version and the compatibility of older versions of Windows for older programs to run smoothly on the recent version of Windows. The "backward compatibility" that helps maximize the ability of the recent version of Windows. The recent version of Windows also can switch device modes as most devices have a tablet mode with a touch screen feature. Windows can adapt to that mode and switch accordingly without issue.

With the constant development of technology today, the companies that develop the operating systems for these devices must keep up with the releases of new devices so that the companies can max out the market's user experience.

\section{RECOMMENDATIONS}

Based on the results gathered by the group, each user interface has its advantages over the other. For example, boot times are faster in Windows 8 and 10, unlike Windows 7. However, Windows 7 is more reliable, has better file transfer rates, and has a better image resolution. Based on the data that the group has gathered, Windows 10 is the fastest version of the Windows user interface and operating system, followed by Windows 8 then Windows 7. Then, in terms of RAM usage, Windows 8 consumes the most RAM, followed by Windows 10 then Windows 7. This means that Windows 10 is the best user interface as compared to the other interfaces such as Windows 8 and Windows 7 . Windows 10 has the fastest processing speed. It is also the most updated in terms of features and security. Moreover, it is the version of Windows that is currently being supported, which means that updates will come regularly. Using all of these points, the group recommends that a user should be using Windows 10 as their operating system. All of the points above are important, as they affect the overall user experience. If the version of Windows being used is not the most recent, the user's data may be more vulnerable to viruses or attacks from downloading software from the internet. This is because of the updates having more security features as vulnerabilities in the operating system are found by Microsoft or by other users. If the user is running an older version of the operating system, it is best to update it, as support for the older operating system and will be removed in the future. By doing so, the user will be able to experience the best possible version of the Windows user interface that he can experience without having to worry too much about vulnerabilities found, as these can be patched out with another update.

\section{REFERENCES}

[1] V. Beal, "The History of Windows Operating Systems," Webopedia. [Online]. Available: https://www.webopedia.com/DidYouKnow/Hardware_S oftware/history_of_microsoft_windows_operating_syste m.html. [Accessed: 06-Aug-2019].

[2] M. Rouse, "What is user interface (UI)? - Definition from WhatIs.com," SearchMicroservices, Nov-2016. [Online]. Available: https://searchmicroservices.techtarget.com/definition/use r-interface-UI. [Accessed: 06-Aug-2019].

[3] D. Lucke, F. Steimle, E. Cuk, M. Luckert, M. Schneider, and D. Schel, "Implementation of the MIALinx User Interface for Future Manufacturing Environments", Procedia CIRP. Vol. 81, No. 1, pp. 606-611, 2019. https://doi.org/10.1016/j.procir.2019.03.163

[4] K. Virtudez, and R. Gustilo, "FPGA implementation of a one-way hash function utilizing HL11-1111 nonlinear digital to analog converter," IEEE Region 10 Annual International Conference, Proceedings/TENCON, 2012. https://doi.org/10.1109/TENCON.2012.6412237

[5] N. J. C. Libatique, G. L. Tangonan, R. Gustilo, W. K. G. Seah, C. Pineda, M. L. Guico, G. Abrajano, R. Ching, J. L. Zamora, A. Espinosa, A. C. Valera, R. Lamac, H. Dy, J. Pusta, E. M. Trono, A. Gimpaya, J. R. S. Luis, S. J. Gonzales and A. T. Lotho, "Design of a tropical rain Disaster alarm system: A new approach based on wireless sensor networks and acoustic rain rate measurements," IEEE Instrum. Meas. Technol. Conf. (I2MTC), pp. 1341-1345, 2009. https://doi.org/10.1109/IMTC.2009.5168663

[6] A. De Albuquerque and J. Kenler, "Toy user interfaces: Systematic and industrial mapping," Journal of Systems Architecture. Vol. 97, No.1, pp. 77-106, 2019.

[7] Y. Shi and Q. Peng, "A VR-based user interface for the upper limb rehabilitation,” Procedia CIRP. Vol 78, No. 1, pp. 115-120, 2018. https://doi.org/10.1016/j.procir.2018.08.311

[8] E. Redondo, D. Fonsesca, A. Sanchez, and I. Navarro, "New Strategies Using Handheld Augmented Reality and Mobile Learning-teaching Methodologies, in Architecture and Building Engineering Degrees," Procedia Computer Science. Vol 25, No. 1, pp. 52-61, 2013.

[9] D. Hepperle, Y. Weib, A. Siess, and M. Wolfel, "2D, 3D or speech? A case study on which user interface is preferable for what kind of object interaction in immersive virtual reality," Computer \& Graphics. Vol 82, No. 1, pp. 321-333, 2019 https://doi.org/10.1016/j.cag.2019.06.003

[10] S. Kim, M. Nussbaum, and J. Gabbard, "Influences of augmented reality head-worn display type and user interface design on performance and usability in simulated warehouse order picking," Applied Ergonomics. Vol 74, No. 1, pp. 186-193, 2019

[11]S. Lindner, M. Yu, J. Capadona and A. Shoffstall, "A graphical user interface to assess the neuroinflammatory response to intracortical microelectrodes," Journal of 
Aaron Don M. Africa et al., International Journal of Emerging Trends in Engineering Research, 8(5), May 2020, 1926 - 1931

Neuroscience Methods. Vol. 317, No. 1, pp. 141-148, 2019.

https://doi.org/10.1016/j.jneumeth.2019.01.003

[12]M. Bockbrader, G. Francisco, R. Lee, J. Olson, R. Solinsky, and M. Boninger, "Brain Computer Interfaces in Rehabilitation Medicine," PM\&R. Vol. 10, No. 1, pp. S223-S243, 2018.

[13] V. Manghisi, U. Antonio, M. Fiorentino, M. Gattullo, A. Boccaccio, and G. Monno, "Enhancing user engagement through the user centric design of a mid-air gesture-based interface for the navigation of virtual-tours in cultural heritage expositions," Journal of Cultural Heritage. Vol. 32, No. 1, pp. 186-197, 2018.

[14] P. Sugathan and K. Ranjan, "Co-creating the tourism experience," Journal of Business Research. Vol. 100, No. 1, pp. 207-217, 2019.

[15] R. Tavakoli and S. Wijesinghe, "The evolution of the web and netography in tourism: A systematic review," Tourism Management Perspectives. Vol. 29, No. 1, pp. 48-55, 2019. https://doi.org/10.1016/j.tmp.2018.10.008

[16] C. Vyas, "Evaluating state tourism using Search Engine Optimization tools," Tourism Management. Vol. 73, No.1, pp. 64-70, 2019.

[17] V. Bogicevic, S. Seo, J. Kandampully, S. Liu, and N. Rudd, "Virtual reality presence as a preamble of tourism experience: The role of mental imagery," Tourism Management. Vol. 74, No.1, pp. 55-64, 2019. https://doi.org/10.1016/j.tourman.2019.02.009

[18]L. Torrizo and A. Africa, "Next-hour electrical load forecasting using an artificial neural network: Applicability in the Philippines," International Journal of Advanced Trends in Computer Science and Engineering. Vol. 8, No. 3, pp. 831-835, 2019. https://doi.org/10.30534/ijatcse/2019/77832019

[19] A. Africa, G. Ching, K. Go, R. Evidente, and J. Uy, “A comprehensive study on application development software systems," International Journal of Emerging Trends in Engineering Research. Vol. 7, No. 8, pp. 99-103, 2019.

https://doi.org/10.30534/ijeter/2019/03782019

[20] A. Africa, F. Espiritu, C. Lontoc, and R. Mendez, "The integration of computer systems into the expansive field of video games," International Journal of Advanced Trends in Computer Science and Engineering. Vol. 8, No. 4, pp. 1139-1145, 2019. https://doi.org/10.30534/ijatcse/2019/22842019

[21] P. Longstreet, X. Xiao, and S. Sarker, "Computer-Related Task Performance: A new perspective," Information \& Management. Vol. 53, No. 4, pp. 517-527, 2016.

[22]A. Ould Baba Alweimine, O. Bamaarouf, A. Rachadi, and H. Ez-Zahraouy, "Local routing protocols performance for computer virus elimination in complex networks," Physica A: Statistical Mechanics and its Applications. Vol.1, No.1, 120984, 2019. https://doi.org/10.1016/j.physa.2019.04.220

[23]I. Chen, "Computer self-efficacy, learning performance, and the mediating role of learning engagement,"
Computers in Human Behavior. Vol. 72, No.1, pp. 362-370, 2017.

[24]L. Morra, N. Coccia, and T. Cerquitelli, "Optimization of computer aided detection systems: An evolutionary approach," Expert Systems with Applications. Vol. 100, No. 1, pp. 145-156, 2018 https://doi.org/10.1016/j.eswa.2018.01.028

[25] H. Saber, S. AlShehri, and W. Maref, "Performance optimization of cascaded and non-cascaded thermoelectric devices for cooling computer chips," Energy Conversion and Management. Vol. 1, No. 1, 2019

[26] A. Ghandar, Z. Michalewicz, and R. Zurbruegg, "The relationship between model complexity and forecasting performance for computer intelligence optimization in finance," International Journal of Forecasting. Vol. 1, No. 1,2016 https://doi.org/10.1016/j.ijforecast.2015.10.003

[27] S. Zhang, H. Ramaswamy, and S. Wang, "Computer simulation modelling, evaluation and optimisation of radio frequency ( $R F$ ) heating uniformity for peanut pasteurisation process," Biosystems Engineering. Vol. 1, No. 1, 2019.

[28]F. Fallani and D. Bassett, "Network neuroscience for optimizing brain-computer interfaces," Physics of Life Reviews. Vol. 1, No. 1, 2019.

[29] I. Tsiptsis, L. Liimatainen, T. Kotnik, and J. Niiranen, "Structural optimization employing isogeometric tools in Particle Swarm Optimizer," Journal of Building Engineering. Vol. 24, No.1, 100761, 2019.

[30]A. Yimit, K. Iigura and Y. Hagihara, "Redefined Selfish Herd Optimizer for Global Optimization Problems," Expert Systems with Applications. Vol.1, No.1, 112838, 2019. https://doi.org/10.1016/j.eswa.2019.112838

[31]A. Africa, P. Arevalo, A. Publico, and M. Tan, "A comprehensive study of the functions and operations of control systems," International Journal of Advanced Trends in Computer Science and Engineering. Vol. 8, No. 3, pp. 922-926, 2019. https://doi.org/10.30534/ijatcse/2019/89832019

[32] A. Africa, P. Arevalo, A. Publico, M. Tan, "Linear system interconnections, steady-state analysis and stability theory." International Journal of Advanced Trends in Computer Science and Engineering. Vol. 8, No. 4, pp 1395-1398, 2019. https://doi.org/10.30534/ijatcse/2019/56842019

[33]A. Africa, "A rough set-based data model for heart disease diagnostics." ARPN Journal of Engineering and Applied Sciences. Vol. 11, No. 15, pp 9350-9357, 2016.

[34]C. Uy and A. Africa, "Development of a cost-efficient waste bin management system with mobile monitoring and tracking." International Journal of Advanced Trends in Computer Science and Engineering. Vol. 8, No. 2, pp 319-327, 2019. https://doi.org/10.30534/ijatcse/2019/35822019

[35]A. Africa, T. Dolores, M. Lim, L. Miguel, and V. Sayoc. "Understanding logical reasoning through computer systems." International Journal of Emerging Trends in Engineering Research. Vol. 8, No. 4, pp 1187-1191, 2020. 